\title{
General Family of Third Order Methods for Multiple Roots of Nonlinear Equations and Basin Attractors for Various Methods
}

\author{
Rajni Sharma ${ }^{1}$ and Ashu Bahl ${ }^{2}$ \\ ${ }^{1}$ Department of Applied Sciences, D.A.V. Institute of Engineering and Technology, Kabir Nagar, Jalandhar 144008, India \\ ${ }^{2}$ Department of Mathematics, D.A.V. College, Jalandhar 144008, India \\ Correspondence should be addressed to Rajni Sharma; rajni_daviet@yahoo.com
}

Received 29 November 2013; Accepted 8 February 2014; Published 31 March 2014

Academic Editor: Ting-Zhu Huang

Copyright (c) 2014 R. Sharma and A. Bahl. This is an open access article distributed under the Creative Commons Attribution License, which permits unrestricted use, distribution, and reproduction in any medium, provided the original work is properly cited.

A general scheme of third order convergence is described for finding multiple roots of nonlinear equations. The proposed scheme requires one evaluation of $f, f^{\prime}$, and $f^{\prime \prime}$ each per iteration and contains several known one-point third order methods for finding multiple roots, as particular cases. Numerical examples are included to confirm the theoretical results and demonstrate convergence behavior of the proposed methods. In the end, we provide the basins of attraction for some methods to observe their dynamics in the complex plane.

\section{Introduction}

Solving nonlinear equations is one of the most important problems in numerical analysis $[1,2]$. With the advancement of computers, the problem of solving nonlinear equations by numerical methods has gained more importance than before. A large number of such methods have been derived for simple roots. However, not many methods are known for multiple root case. In this paper, we consider iterative methods to find a multiple root $\alpha$ of multiplicity $m>1$, that is, $f^{(j)}(\alpha)=0$, $j=0,1, \ldots m-1$, and $f^{(m)}(\alpha) \neq 0$, of a nonlinear equation $f(x)=0$, where $f(x)$ is the continuously differentiable real or complex function. The modified Newton method [3] is an important and basic method for finding multiple roots

$$
x_{k+1}=x_{k}-m \frac{f\left(x_{k}\right)}{f^{\prime}\left(x_{k}\right)}
$$

which converges quadratically and requires the knowledge of multiplicity $m$ of root $\alpha$.

In order to improve the order of convergence of (1), several third order methods with known multiplicity $m$ have been proposed at the expense of an additional evaluation of second derivative (see [4-14]).

Inspired by the work in this direction, here we present a third order scheme of one-point methods without memory for multiple roots. The proposed scheme involves one evaluation of $f, f^{\prime}$, and $f^{\prime \prime}$ each per step. It is also shown that the well known existing methods by Ostrowski [4], Hansen and Patrick [5], Traub [6], Neta [8], Osada [7], Chun et al. [9, 10], Biazar and Ghanbari [11], and J. R. Sharma and R. Sharma $[12,13]$ can be regarded as particular cases of the proposed family.

The paper is organized in six sections. In Section 2, the third order family of methods for multiple roots requiring one evaluation of $f, f^{\prime}$, and $f^{\prime \prime}$ each per step is proposed and its convergence behavior is discussed. Some particular cases of the family are presented in Section 3. In Section 4, the new methods are compared with the closest competitors in a series of numerical examples. In Section 5, we investigate the basins of attraction for some of the methods to provide the chaotic behavior of such schemes. Concluding remarks are given in Section 6.

\section{The Method and Its Convergence Analysis}

Consider the following iterative scheme:

$$
x_{k+1}=x_{k}-H\left(\frac{f\left(x_{k}\right) f^{\prime \prime}\left(x_{k}\right)}{f^{\prime}\left(x_{k}\right)^{2}}\right) \frac{f\left(x_{k}\right)}{f^{\prime}\left(x_{k}\right)},
$$


where the function $H(\cdot) \in C^{2}(\mathbb{R})$. In order to discuss the properties of the scheme defined by (2), we prove the following theorem.

Theorem 1. Let $\alpha \in I$ be a multiple root of multiplicity $m$ of a sufficiently differentiable function $f: I \rightarrow \mathbb{R}$ for an open interval I. If $x_{0}$ is sufficiently close to $\alpha$, then the iterative scheme defined by (2) has third order convergence, provided $H(u)=m, H^{\prime}(u)=m^{2} / 2$, and $H^{\prime \prime}(u)<\infty$, and for $u=(m-1) / m, e_{k}=x_{k}-\alpha$, one has

$$
e_{k+1}=\left[\frac{m^{4}+3 m^{3}-4 H^{\prime \prime}(u)}{2 m^{5}} A_{1}^{2}-\frac{A_{2}}{m}\right] e_{k}^{3}+O\left(e_{k}^{4}\right),
$$

where $A_{i}=m ! f^{(m+i)}(\alpha) /(m+i) ! f^{(m)}(\alpha)$, for $i \in \mathbb{N}, \mathbb{N}$ being the set of natural numbers.

Proof. Expand $f\left(x_{k}\right), f^{\prime}\left(x_{k}\right), f^{\prime \prime}\left(x_{k}\right)$ in the Taylor series about $\alpha$, where $\alpha$ is a multiple root of multiplicity $m$. Thus using the fact that $f^{(j)}(\alpha)=0, j=0,1, \ldots m-1$, and $f^{(m)}(\alpha) \neq 0$, we have

$$
\begin{aligned}
f\left(x_{k}\right) & =\frac{f^{(m)}(\alpha) e_{k}^{m}}{m !}\left[1+A_{1} e_{k}+A_{2} e_{k}^{2}+A_{3} e_{k}^{3}+O\left(e_{k}^{4}\right)\right], \\
f^{\prime}\left(x_{k}\right) & =\frac{f^{(m)}(\alpha) e_{k}^{m-1}}{(m-1) !}\left[1+B_{1} e_{k}+B_{2} e_{k}^{2}+B_{3} e_{k}^{3}+O\left(e_{k}^{4}\right)\right], \\
f^{\prime \prime}\left(x_{k}\right) & =\frac{f^{(m)}(\alpha) e_{k}^{m-2}}{(m-2) !}\left[1+C_{1} e_{k}+C_{2} e_{k}^{2}+C_{3} e_{k}^{3}+O\left(e_{k}^{4}\right)\right],
\end{aligned}
$$

where

$$
\begin{gathered}
A_{i}=\frac{m ! f^{(m+i)}(\alpha)}{(m+i) ! f^{(m)}(\alpha)}, \quad B_{i}=\frac{(m-1) ! f^{(m+i)}(\alpha)}{(m+i-1) ! f^{(m)}(\alpha)}, \\
C_{i}=\frac{(m-2) ! f^{(m+i)}(\alpha)}{(m+i-2) ! f^{(m)}(\alpha)} \\
i=1,2, \ldots
\end{gathered}
$$

From (4), we get

$$
\begin{aligned}
\frac{f\left(x_{k}\right)}{f^{\prime}\left(x_{k}\right)}= & \frac{e_{k}}{m}-\frac{A_{1} e_{k}^{2}}{m^{2}}+\frac{1}{m^{3}}\left[(m+1) A_{1}^{2}-2 m A_{2}\right] e_{k}^{3} \\
& +O\left(e_{k}^{4}\right),
\end{aligned}
$$

$$
\begin{aligned}
\frac{f\left(x_{k}\right) f^{\prime \prime}\left(x_{k}\right)}{f^{\prime}\left(x_{k}\right)^{2}}= & \frac{m-1}{m}+\frac{2 A_{1} e_{k}}{m^{2}} \\
& -\frac{3}{m^{3}}\left[(m+1) A_{1}^{2}-2 m A_{2}\right] e_{k}^{2} \\
& +\frac{4}{m^{4}}\left[(m+1) A_{1}^{3}-m(3 m+4) A_{1} A_{2}\right. \\
& \left.+3 m^{2} A_{3}\right] e_{k}^{3}+O\left(e_{k}^{4}\right) .
\end{aligned}
$$

Let $f\left(x_{k}\right) f^{\prime \prime}\left(x_{k}\right) / f^{\prime}\left(x_{k}\right)^{2}=u+v$, where $u=(m-1) / m$. Then from (7), the remainder $v=\left(f\left(x_{k}\right) f^{\prime \prime}\left(x_{k}\right) / f^{\prime}\left(x_{k}\right)^{2}\right)-u$ is infinitesimal with the same order of $e_{k}$.

Thus we can use the Taylor series to expand $H\left(f\left(x_{k}\right) f^{\prime \prime}\left(x_{k}\right) / f^{\prime}\left(x_{k}\right)^{2}\right)=H(u+v)$ about $u$ and then obtain

$$
\begin{aligned}
H\left(\frac{f\left(x_{k}\right) f^{\prime \prime}\left(x_{k}\right)}{f^{\prime}\left(x_{k}\right)^{2}}\right)= & H(u)+H^{\prime}(u) v+\frac{H^{\prime \prime}(u) v^{2}}{2} \\
& +\frac{H^{\prime \prime \prime}(u) v^{3}}{3 !}+O\left(e_{k}^{4}\right) .
\end{aligned}
$$

By invocation of (2), (6), and (8), we get the error equation as

$$
e_{k+1}=K_{1} e_{k}+K_{2} e_{k}^{2}+K_{3} e_{k}^{3}+O\left(e_{k}^{4}\right),
$$

where

$$
\begin{aligned}
& K_{1}=1-\frac{H(u)}{m}, \quad K_{2}=\frac{H(u) m-2 H^{\prime}(u)}{m^{3}} A_{1}, \\
& K_{3}=-\frac{1}{m^{5}}[\left(\left(m^{3}+m^{2}\right) H(u)\right. \\
&\left.-\left(3 m^{2}+5 m\right) H^{\prime}(u)+2 H^{\prime \prime}(u)\right) A_{1}^{2} \\
&\left.+\left(6 m^{2} H^{\prime}(u)-2 m^{3} H(u)\right) A_{2}\right] .
\end{aligned}
$$

In order to achieve the third order convergence, the coefficients $K_{1}$ and $K_{2}$ must vanish. Solving $K_{1}=0$ and $K_{2}=0$, we obtain

$$
H(u)=m, \quad H^{\prime}(u)=\frac{m^{2}}{2} .
$$

Hence, (9) becomes

$$
e_{k+1}=\left[\frac{m^{4}+3 m^{3}-4 H^{\prime \prime}(u)}{2 m^{5}} A_{1}^{2}-\frac{A_{2}}{m}\right] e_{k}^{3}+O\left(e_{k}^{4}\right) .
$$

This completes the proof of Theorem 1 .

Remark 2. From the error equation (9), we can find that the iterative method (2) contains the modified Newton method (1) as a second order method also.

Remark 3. Obviously, the number of function evaluations per iteration required in the scheme defined by (2) is three. We consider the definition of efficiency index [15] as $p^{1 / r}$ where $p$ is the order of the method and $r$ is the number of function evaluations per iteration required by the method. The scheme defined by (2) has the efficiency index equal to $3^{1 / 3} \approx 1.442$ which is much better than $2^{1 / 2} \approx 1.414$ of the modified Newton method.

\section{Some Special Cases of Order Three}

In this section, we present some special cases of order three of the presented scheme (2). 
Case 1. For the function $H$ defined by $H(t)=A t+B, H^{\prime}(t)=$ A.

According to (11), solving the equations

$$
A u+B=m, \quad A=\frac{m^{2}}{2},
$$

we get $A=m^{2} / 2, B=m(3-m) / 2$. With these values of $A$ and $B$, scheme (2) takes the form

$$
x_{k+1}=x_{k}-m\left[\frac{m}{2} \frac{f\left(x_{k}\right) f^{\prime \prime}\left(x_{k}\right)}{f^{\prime}\left(x_{k}\right)^{2}}+\frac{3-m}{2}\right] \frac{f\left(x_{k}\right)}{f^{\prime}\left(x_{k}\right)},
$$

which is Chebyshev's method for multiple roots, proposed in $[6,8]$.

Case 2. If $H(t)=A+(B / t)$, then $H^{\prime}(t)=-B / t^{2}$.

According to (11), solving the equations

$$
A+\frac{B}{u}=m, \quad-\frac{B}{u^{2}}=\frac{m^{2}}{2}
$$

we get $A=m(m+1) / 2, B=-(m-1)^{2} / 2$. With these values, scheme (2) leads to

$$
\begin{aligned}
x_{k+1}= & x_{k}-\left[\frac{m(m+1)}{2}-\frac{(m-1)^{2}}{2} \frac{f^{\prime}\left(x_{k}\right)^{2}}{f\left(x_{k}\right) f^{\prime \prime}\left(x_{k}\right)}\right] \\
& \times \frac{f\left(x_{k}\right)}{f^{\prime}\left(x_{k}\right)},
\end{aligned}
$$

which is the third order Osada method for multiple roots [7].

Case 3. Taking $H(t)=1 /(A t+B)$, then $H^{\prime}(t)=-A /(A t+B)^{2}$.

According to (11), solving the equations

$$
\frac{1}{A u+B}=m, \quad-\frac{A}{(A u+B)^{2}}=\frac{m^{2}}{2}
$$

we get $A=-1 / 2$ and $B=(m+1) / 2 m$. With these values, (2) yields

$$
\begin{aligned}
x_{k+1}=x_{k}-f\left(x_{k}\right) \times & \left(((m+1) /(2 m)) f^{\prime}\left(x_{k}\right)\right. \\
& \left.-\left(\left(f\left(x_{k}\right) f^{\prime \prime}\left(x_{k}\right)\right) /\left(2 f^{\prime}\left(x_{k}\right)\right)\right)\right)^{-1},
\end{aligned}
$$

which is Halley's method for multiple roots [5].

Case 4. Supposing that $H(t)=1 / \sqrt{A+B t}$, then $H^{\prime}(t)=$ $-B / 2(A+B t)^{3 / 2}$.

According to (11), solving the equations

$$
\frac{1}{\sqrt{A+B u}}=m, \quad-\frac{B}{2(A+B u)^{3 / 2}}=\frac{m^{2}}{2},
$$

we get $A=1 / m$ and $B=-1 / m$ and hence (2) becomes

$$
x_{k+1}=x_{k}-\left[\frac{\sqrt{m}}{\sqrt{1-\left(f\left(x_{k}\right) f^{\prime \prime}\left(x_{k}\right)\right) / f^{\prime}\left(x_{k}\right)^{2}}}\right] \frac{f\left(x_{k}\right)}{f^{\prime}\left(x_{k}\right)},
$$

which is Ostrowski's square root iteration [4].

Case 5. If $H(t)=(A+B t) /(C+D t)$, we have $H^{\prime}(t)=(B C-$ $A D) /(C+D t)^{2}$.

According to (11), solving the equations

$$
\frac{A+B u}{C+D u}=m, \quad \frac{B C-A D}{(C+D u)^{2}}=\frac{m^{2}}{2}
$$

we get

$$
A=\frac{C m(3-m)-D(m-1)^{2}}{2}, \quad B=\frac{D m+(C+D) m^{2}}{2} .
$$

Subcase 1. The choice of $C=2$ and $D=-\beta(\beta \in \mathbb{R})$ gives

$$
A=\frac{2 m(3-m)+\beta(m-1)^{2}}{2}, \quad B=-\frac{\beta m+(\beta-2) m^{2}}{2} \text {. }
$$

With these values, (2) corresponds to the Sharma-Sharma method [13]:

$$
\begin{aligned}
& x_{k+1} \\
& =x_{k}-\frac{1}{4} \\
& \times\left[\beta(m-1)^{2}-2 m(m-3)\right. \\
& \left.+\frac{(\beta(m-1)-2 m)^{2}\left(f\left(x_{k}\right) f^{\prime \prime}\left(x_{k}\right) / 2 f^{\prime}\left(x_{k}\right)^{2}\right)}{1-\beta\left(f\left(x_{k}\right) f^{\prime \prime}\left(x_{k}\right) / 2 f^{\prime}\left(x_{k}\right)^{2}\right)}\right] \\
& \times \frac{f\left(x_{k}\right)}{f^{\prime}\left(x_{k}\right)} \\
& x_{k+1}=x_{k} \\
& -\frac{2 m^{2} f\left(x_{k}\right)^{2} f^{\prime \prime}\left(x_{k}\right)}{m(3-m) f\left(x_{k}\right) f^{\prime}\left(x_{k}\right) f^{\prime \prime}\left(x_{k}\right)+(m-1)^{2} f^{\prime}\left(x_{k}\right)^{3}},
\end{aligned}
$$

which is the third order Chun-Neta method [9]. 
Case 6. For

$$
\begin{gathered}
H(t)=\frac{\left(A+B t+C t^{2}\right)}{\left(D+E t+F t^{2}\right)}, \\
H^{\prime}(t)=\frac{(B D-A E)-2(A F-C D) t+(C E-B F) t^{2}}{\left(D+E t+F t^{2}\right)^{2}} .
\end{gathered}
$$

According to (11), solving the equations $\left(A+B u+C u^{2}\right) /(D+$ $\left.E u+F u^{2}\right)=m$ and

$$
\frac{(B D-A E)-2(A F-C D) u+(C E-B F) u^{2}}{\left(D+E u+F u^{2}\right)^{2}}=\frac{m^{2}}{2},
$$

we get

$$
\begin{aligned}
E= & \frac{C(m-1)^{3}+B m\left(m^{2}-1\right)+m^{2}(A(m+3)-4 D m)}{2 m^{2}(m-1)} \\
F= & -\frac{C(m-3)(m-1)^{2}+B m(m-1)^{2}+m^{2}(A(m+1))}{2 m(m-1)^{2}} \\
& +\frac{2 D m^{3}}{2 m(m-1)^{2}} .
\end{aligned}
$$

Subcase 1. Taking $D=0$ and $E=1$ and solving (28), we get

$$
\begin{gathered}
A=-\frac{(m-1)^{2}\left((F+1) m^{2}+F m-2 C\right)}{2 m^{2}}, \\
B=\frac{m^{2}(F m+m+2 F+1)-4 C m-3 F m+4 C}{2 m} .
\end{gathered}
$$

With these values, (2) corresponds to the third order family of multiple roots developed by Biazar and Ghanbari [11]:

$$
\begin{aligned}
x_{k+1}=x_{k}-( & \left(B f\left(x_{k}\right) f^{\prime}\left(x_{k}\right)^{2} f^{\prime \prime}\left(x_{k}\right)\right. \\
& \left.+A f^{\prime}\left(x_{k}\right)^{4}+C f\left(x_{k}\right)^{2} f^{\prime \prime}\left(x_{k}\right)^{2}\right) \\
& \times\left(f^{\prime}\left(x_{k}\right)^{3} f^{\prime \prime}\left(x_{k}\right)\right. \\
& \left.\left.+F f\left(x_{k}\right) f^{\prime}\left(x_{k}\right) f^{\prime \prime}\left(x_{k}\right)^{2}\right)^{-1}\right),
\end{aligned}
$$

where $A$ and $B$ are given in (29).
Subcase 2. Taking $A=2\left(m^{2}-3 m+2\right), B=-m^{2}+5 m, C=$ $3 m^{2}$, and $D=2 m^{2}-6 m+4,(28)$ gives $E=-2\left(m^{2}-4 m+1\right)$ and $F=0$. With these values, (2) yields

$$
\begin{aligned}
x_{k+1}=x_{k}-[1 & +\frac{f\left(x_{\mathrm{k}}\right) f^{\prime \prime}\left(x_{k}\right)}{2 f^{\prime}\left(x_{k}\right)^{2}} \\
& +\left((2 m-1)^{2}\left(f\left(x_{k}\right) f^{\prime \prime}\left(x_{k}\right) / f^{\prime}\left(x_{k}\right)^{2}\right)^{2}\right) \\
& \times\left(2 \left(\left(m^{2}-3 m+2\right)-\left(m^{2}-4 m+1\right)\right.\right. \\
& \left.\left.\left.\times\left(f\left(x_{k}\right) f^{\prime \prime}\left(x_{k}\right) / f^{\prime}\left(x_{k}\right)^{2}\right)\right)\right)^{-1}\right] \\
& \times \frac{f\left(x_{k}\right)}{f^{\prime}\left(x_{k}\right)},
\end{aligned}
$$

which is the Sharma-Sharma method [12].

Subcase 3. With $\theta \in \mathbb{R}$, taking

$$
\begin{gathered}
A=-\frac{\theta(m-1)^{2}}{2}, \quad B=\frac{(2 \theta-1) m^{2}+(3-2 \theta) m}{2}, \\
C=\frac{(1-\theta) m^{2}}{2} .
\end{gathered}
$$

and $D=0,(28)$ generates $E=1$ and $F=0$. So, (2) leads to

$$
\begin{aligned}
x_{k+1}= & x_{k} \\
- & {\left[\frac{m[(2 \theta-1) m+3-2 \theta]}{2}\right.} \\
& -\frac{\theta(m-1)^{2}}{2} \frac{f^{\prime}\left(x_{k}\right)^{2}}{f\left(x_{k}\right) f^{\prime \prime}\left(x_{k}\right)} \\
& \left.+\frac{(1-\theta) m^{2}}{2} \frac{f\left(x_{k}\right) f^{\prime \prime}\left(x_{k}\right)}{f^{\prime}\left(x_{k}\right)^{2}}\right] \frac{f\left(x_{k}\right)}{f^{\prime}\left(x_{k}\right)},
\end{aligned}
$$

which is the one-parameter family of methods for multiple roots proposed by Chun et al. [10].

Case 7. Further, if $H(t)=A+(B / t)+\left(C / t^{2}\right)$, then $H^{\prime}(t)=$ $-\left(B / t^{2}\right)-\left(2 C / t^{3}\right)$.

According to (11), solving the equations

$$
A+\frac{B}{u}+\frac{C}{u^{2}}=m, \quad-\frac{B}{u^{2}}-\frac{2 C}{u^{3}}=\frac{m^{2}}{2},
$$

we get $A=\left(\left(m+m^{2}\right) / 2\right)+\left(C m^{2} /(m-1)^{2}\right)$ and $B=(-(m-$ $\left.1)^{2} / 2\right)-(2 \mathrm{Cm} /(m-1))$. 
TABLE 1: Test functions.

\begin{tabular}{llr}
\hline$f(x)$ & $\alpha$ & $m$ \\
\hline$f_{1}(x)=\left((x-1)^{3}-1\right)^{6}$ & 2.0000000000000000000000000000 & 6 \\
$f_{2}(x)=\left(\ln \left(x^{2}+x+2\right)-x+1\right)^{4}$ & 4.1525907367571582749969890048 & 4 \\
$f_{3}(x)=\left(\ln ^{2}(x-2)\left(e^{x-3}-1\right) \sin \frac{\pi x}{3}\right)$ & 3.0000000000000000000000000000 & 4 \\
$f_{4}(x)=\left(x^{2}-e^{x}-3 x+2\right)^{5}$ & 0.2575302854398607604553673049 & 5 \\
$f_{5}(x)=(\cos x-x)^{3}$ & 0.73908513321516064165531208767 & 3 \\
$f_{6}(x)=\left(\sqrt{ } x-\frac{1}{x}-3\right)^{3}$ & 9.6335955628326951924063127092 & 3 \\
$f_{7}(x)=\left(x^{3}-10\right)^{8}$ & 2.1544346900318837217592935665 & \\
\hline
\end{tabular}

TABLe 2: Performance of the methods using 12 NFE.

\begin{tabular}{cccccccc}
\hline \multirow{2}{*}{$f(x)$} & $x_{0}$ & MNM & OM & HM & SSM $(\beta=3 / 2)$ & CNM & SBM $(C=1 / 2)$ \\
\hline \multirow{2}{*}{$f_{1}$} & 1.75 & $1.20(-34)$ & $2.76(-22)$ & $5.48(-34)$ & $4.01(-41)$ & $1.08(-42)$ & $1.25(-22)$ \\
& 3 & $1.55(-16)$ & $2.75(-15)$ & $4.93(-18)$ & $2.36(-20)$ & $6.78(-21)$ & $2.37(-15)$ \\
\hline \multirow{2}{*}{$f_{2}$} & 4 & $4.53(-129)$ & $4.46(-137)$ & $1.46(-145)$ & $1.17(-144)$ & $1.11(-141)$ & $1.75(-137)$ \\
& 5 & $6.88(-86)$ & $2.45(-84)$ & $7.75(-91)$ & $3.78(-90)$ & $7.10(-88)$ & $1.19(-84)$ \\
\hline \multirow{2}{*}{$f_{3}$} & 2.5 & $5.41(-67)$ & $2.47(-76)$ & $8.33(-57)$ & $5.60(-63)$ & $4.90(-58)$ & $1.34(-74)$ \\
& 3.25 & $7.83(-99)$ & $2.32(-118)$ & $1.39(-106)$ & $2.61(-110)$ & $2.60(-107)$ & $2.35(-115)$ \\
\hline \multirow{2}{*}{$f_{4}$} & -2 & $1.55(-37)$ & $1.30(-42)$ & $1.45(-40)$ & $1.52(-39)$ & $1.47(-40)$ & $2.52(-40)$ \\
& 1.5 & $1.06(-57)$ & $2.44(-50)$ & $5.15(-50)$ & $2.43(-40)$ & $4.66(-38)$ & $1.33(-49)$ \\
\hline \multirow{2}{*}{$f_{5}$} & 0.3 & $3.59(-59)$ & $2.13(-44)$ & $5.12(-64)$ & $5.12(-64)$ & $7.17(-53)$ & $1.16(-47)$ \\
& 1.7 & $3.25(-65)$ & $5.35(-41)$ & $2.25(-44)$ & $2.25(-44)$ & $1.84(-42)$ & $1.66(-41)$ \\
\hline \multirow{2}{*}{$f_{6}$} & 9 & $5.13(-108)$ & $2.51(-137)$ & $1.50(-135)$ & $1.50(-135)$ & $1.15(-193)$ & $9.43(-146)$ \\
& 10 & $1.11(-61)$ & $3.59(-158)$ & $4.68(-157)$ & $4.68(-157)$ & $9.07(-214)$ & $7.54(-167)$ \\
\hline \multirow{2}{*}{$f_{7}$} & 2 & $2.59(-72)$ & $1.68(-78)$ & $6.75(-99)$ & $3.89(-89)$ & $3.29(-92)$ & $1.44(-78)$ \\
& 3 & $2.79(-33)$ & $3.19(-34)$ & $2.97(-47)$ & $9.07(-41)$ & $8.73(-43)$ & $2.92(-34)$ \\
\hline
\end{tabular}

Here $a(-b)=a \times 10^{-b}$.

TABLE 3: Computational order of convergence $(\rho)$.

\begin{tabular}{|c|c|c|c|c|c|c|c|}
\hline \multirow{2}{*}{$f(x)$} & \multirow{2}{*}{$x_{0}$} & \multicolumn{6}{|c|}{$\rho$} \\
\hline & & MNM & $\mathrm{OM}$ & $\mathrm{HM}$ & $\operatorname{SSM}(\beta=3 / 2)$ & $\mathrm{CNM}$ & $\operatorname{SBM}(C=1 / 2)$ \\
\hline \multirow{2}{*}{$f_{1}$} & 1.75 & 2 & 3 & 3 & 3 & 3 & 3 \\
\hline & 3 & 2 & 3 & 3 & 2.99 & 2.99 & 3 \\
\hline \multirow{2}{*}{$f_{2}$} & 4 & 2 & 3 & 3 & 3 & 3 & 3 \\
\hline & 5 & 2 & 3 & 3 & 3 & 3 & 3 \\
\hline \multirow{2}{*}{$f_{3}$} & 2.5 & 2 & 3 & 3 & 3 & 3 & 3 \\
\hline & 3.25 & 2 & 3 & 3 & 3 & 3 & 3 \\
\hline \multirow{2}{*}{$f_{4}$} & -2 & 2 & 3 & 3 & 3 & 3 & 3 \\
\hline & 1.5 & 2 & 3 & 3 & 3 & 3 & 3 \\
\hline \multirow{2}{*}{$f_{5}$} & 0.3 & 2 & 3 & 3 & 3 & 3 & 3 \\
\hline & 1.7 & 2 & 2.99 & 2.99 & 2.99 & 2.99 & 3 \\
\hline \multirow{2}{*}{$f_{6}$} & 9 & 2 & 3 & 3 & 3 & 3 & 3 \\
\hline & 10 & 2 & 3 & 3 & 3 & 3 & 3 \\
\hline \multirow{2}{*}{$f_{7}$} & 2 & 2 & 3 & 3 & 3 & 3 & 3 \\
\hline & 3 & 2 & 2.99 & 2.99 & 2.99 & 2.99 & 3 \\
\hline
\end{tabular}




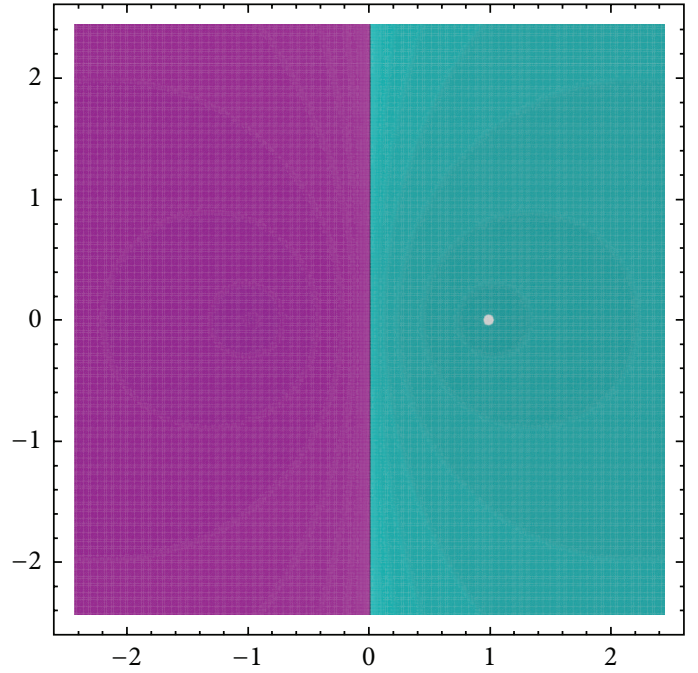

(a) The modified Newton method

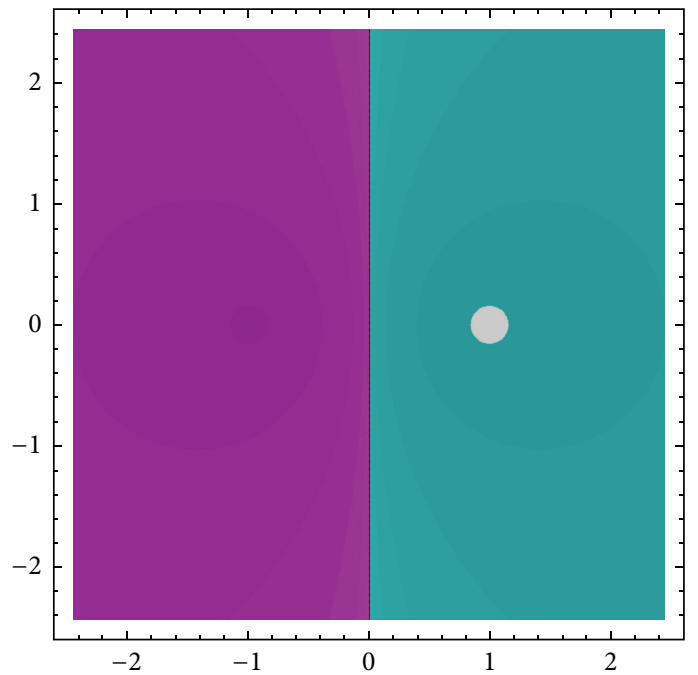

(c) Halley's method

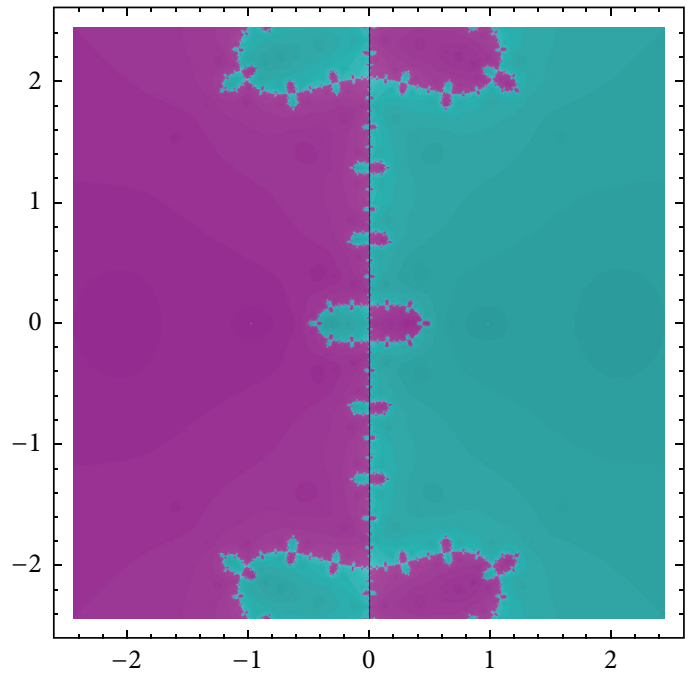

(e) The Sharma-Sharma method $(\beta=3 / 2)$

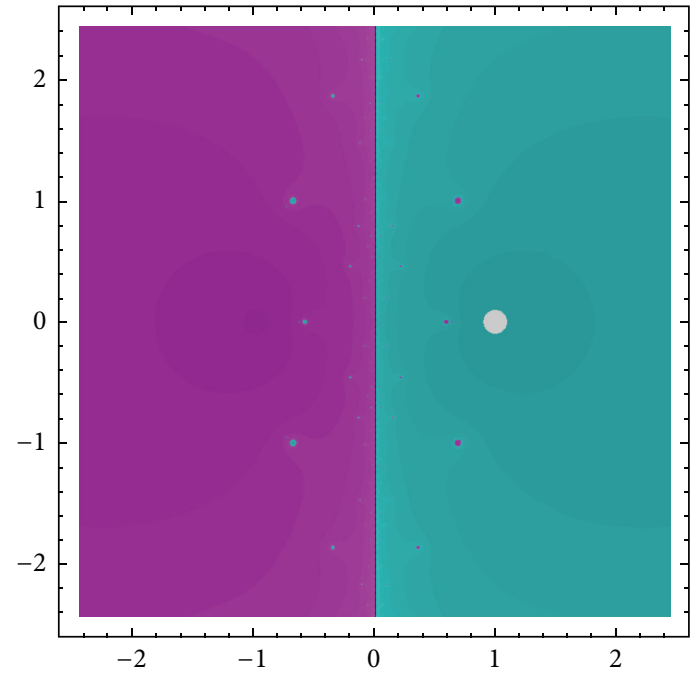

(b) The Osada method

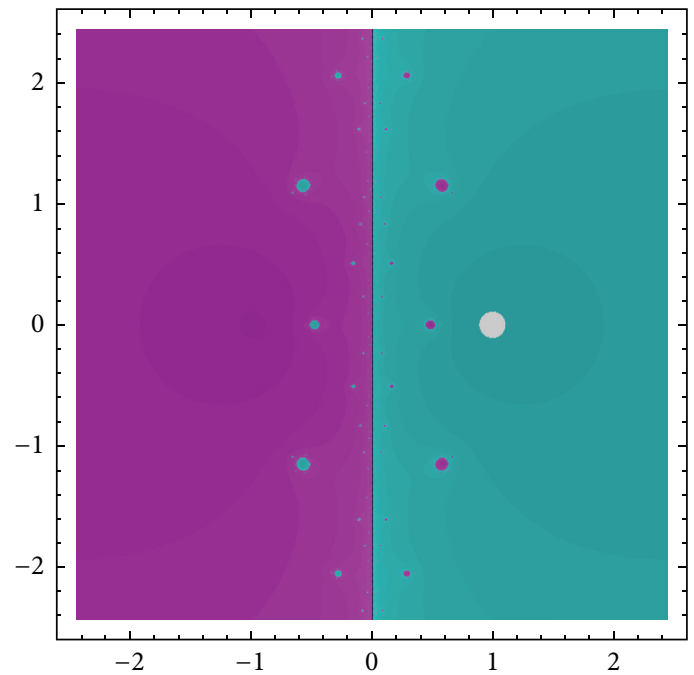

(d) The Chun-Neta method

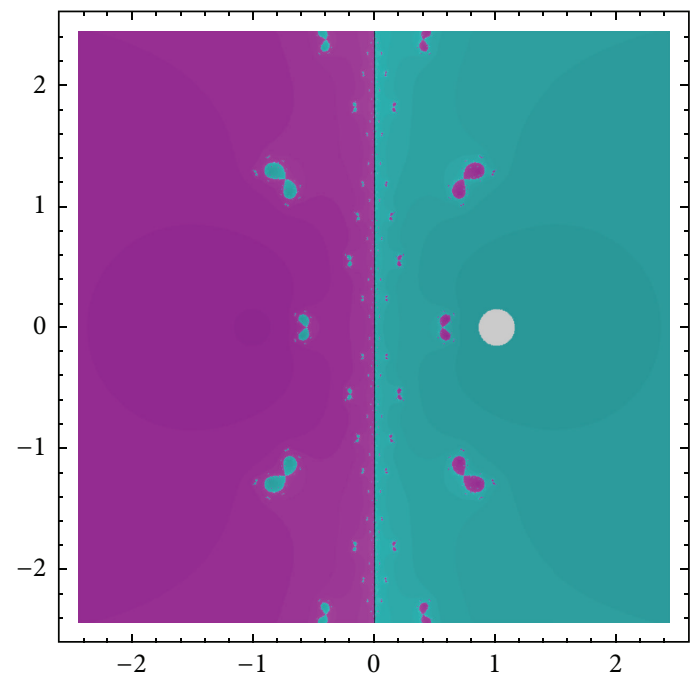

(f) $\operatorname{SBM}(C=1 / 2)$

Figure 1: Basins of attraction for $f(z)=\left(z^{2}-1\right)^{2}, z \in D$, for various methods of the family. 
So, we introduce a new one-parameter third order method as

$$
\begin{aligned}
x_{k+1}=x_{k}- & {\left[\frac{m+m^{2}}{2}+\frac{C m^{2}}{(m-1)^{2}}\right) } \\
& +\left(\frac{-(m-1)^{2}}{2}-\frac{2 C m}{m-1}\right) \frac{f^{\prime}\left(x_{k}\right)^{2}}{f\left(x_{k}\right) f^{\prime \prime}\left(x_{k}\right)} \\
& \left.+C\left(\frac{\left(f^{\prime}\left(x_{k}\right)\right)^{2}}{\left(f\left(x_{k}\right) f^{\prime \prime}\left(x_{k}\right)\right)}\right)^{2}\right] \frac{f\left(x_{k}\right)}{f^{\prime}\left(x_{k}\right)},
\end{aligned}
$$

where $C \in \mathbb{R}$. We call this method the Sharma-Bahl method, denoted by SBM.

\section{Numerical Examples}

In this section, we employ the presented third order method SBM (35) for $C=1 / 2$ to solve some nonlinear equations which not only illustrate the methods practically but also serve to check the validity of the theoretical results we have derived. To check the theoretical order of convergence, we obtain the computational order of convergence $(\rho)$ using the formula [16]

$$
\rho \approx \frac{\ln \left|\left(x_{k+1}-\alpha\right) /\left(x_{k}-\alpha\right)\right|}{\ln \left|\left(x_{k}-\alpha\right) /\left(x_{k-1}-\alpha\right)\right|} .
$$

The performance is compared with the methods in our families including the special ones, for some arbitrary chosen functions with roots of known multiplicity $m$. The methods compared include the modified Newton method (MNM) (1), the Osada method (OM) (16), Halley's method (HM) (18), the Sharma-Sharma method (SSM) (24) for $\beta=3 / 2$, and the Chun-Neta method (CNM) (25).

The test functions along with root $\alpha$ correct up to 28 decimal places and its multiplicity $m$ is displayed in Table 1. Table 2 shows the values of initial approximation $\left(x_{0}\right)$ chosen from both ends to the root and the values of the error $\left|e_{k}\right|=$ $\left|x_{k}-\alpha\right|$ calculated by costing the same total number of function evaluations (NFE) for each method. Table 3 exhibits the computational order of convergence $(\rho)$. For numerical illustrations in Table 3, we use fixed stopping criterion $\epsilon=$ $0.5 \times 10^{-28}$. The NFE is counted as sum of the number of evaluations of the function plus the number of evaluations of the derivatives. We decide to choose 12 NFE for each method. That means that, for MNM, the error $\left|e_{k}\right|$ is calculated at the sixth iteration, whereas for the remaining methods this is calculated at the fourth iteration. All computations are performed by MATHEMATICA [17] using 600 significant digits.

It can also be observed from the numerical results of Table 2 that all the presented methods are well behaved and that, for most of the functions we tested, the method introduced in this paper has similar performance compared to the other methods, as expected from methods of the same order and same computational efficiency. However, Halley's method (18) and the Chun-Neta method (25) behave better and these are special cases of our proposed scheme for linear rational function $H(\cdot)$. The results of Table 3 show that the computational order of convergence is in accordance with the theoretical order of convergence. The formulae have been employed on several other nonlinear equations and results are found at par with those presented here. A reasonably close starting value is necessary for the method to converge. The condition, however, practically applies to all iterative methods for solving nonlinear equations.

\section{Finding the Basins}

In this section, we find the basins of attraction of complex roots for the a complex function. We consider the simplest case $f(z)=\left(z^{2}-1\right)^{2}$, which has roots $z= \pm 1$ of multiplicity 2 . Cayley [18] was the first who considered the Newton method for the roots of polynomial with iterations over the complex numbers.

We take the initial point as $z_{0} \in D$ where $D$ is a rectangular region $[-2.5,2.5] \times[-2.5,2.5] \subset \mathbb{C}$ containing all the roots of $f(z)=0$. We consider the stopping criterion for convergence as $10^{-3}$ up to a maximum of 25 iterations. We take a grid of $1024 \times 1024$ points in $D$.

To generate the pictures, we use MATHEMATICA [17]. We assign the light to dark colors based on the number of iterations in which the considered initial point converges to a root. The root $z=1$ of $f(z)=0$ and the points in the region converging to this root are shaded in cyan color and magenta color is used for the other root $z=-1$ in a similar way. If we have not obtained the desired tolerance in 25 iterations, we do not continue and we decide that the iterative method starting at $z_{0}$ does not converge to any root and such points are shaded in black color (see [19-22]).

The figures given here clearly show that the modified Newton method (Figure 1(a)) and Halley's method (Figure 1(c)) are the best, followed by the Osada method (Figure 1(b)), the Chun-Neta method (Figure 1(d)), SBM (Figure 1(f)), and Sharma-Sharma method (Figure 1(e)). From these pictures, we can guess the behavior and suitability of any method depending upon the circumstances. Thus, concluding, we can say that the linear rational function gives the best choice of weight function $H(\cdot)$.

\section{Conclusions}

In this paper, a general family of one-point third order methods for finding multiple roots of nonlinear equations is presented. This scheme contains some well known methods and recently developed methods as its particular cases. The theoretical results have been checked with some numerical examples, comparing our methods with the modified Newton method and some third order methods. The presented basins of attraction have also demonstrated the strictness of method for choice of starting point. 


\section{Conflict of Interests}

The authors declare that there is no conflict of interests regarding the publication of this paper.

\section{Acknowledgment}

The authors are grateful to the anonymous reviewers for their valuable suggestions and comments on this paper.

\section{References}

[1] J. M. Ortega and W. C. Rheinboldt, Iterative Solution of Nonlinear Equations in Several Variables, Academic Press, New York, NY, USA, 1970.

[2] S. C. Chapra and R. P. Canale, Numerical Methods for Engineers, McGraw-Hill Book Company, New York, NY, USA, 1988.

[3] E. Schröder, "Ueber unendlich viele Algorithmen zur Auflösung der Gleichungen," Mathematische Annalen, vol. 2, no. 2, pp. 317365,1870 .

[4] A. M. Ostrowski, Solution of Equations in Euclidean and Banach Spaces, Academic Press, New York, NY, USA, 3rd edition, 1973.

[5] E. Hansen and M. Patrick, "A family of root finding methods," Numerische Mathematik, vol. 27, no. 3, pp. 257-269, 1977.

[6] J. F. Traub, Iterative Methods for the Solution of Equations, Prentice-Hall, Englewood Cliffs, NJ, USA, 1964.

[7] N. Osada, "An optimal multiple root-finding method of order three," Journal of Computational and Applied Mathematics, vol. 51, no. 1, pp. 131-133, 1994.

[8] B. Neta, "New third order nonlinear solvers for multiple roots," Applied Mathematics and Computation, vol. 202, no. 1, pp. 162$170,2008$.

[9] C. Chun and B. Neta, "A third-order modification of Newton's method for multiple roots," Applied Mathematics and Computation, vol. 211, no. 2, pp. 474-479, 2009.

[10] C. Chun, H. J. Bae, and B. Neta, "New families of nonlinear third-order solvers for finding multiple roots," Computers and Mathematics with Applications, vol. 57, no. 9, pp. 1574-1582, 2009.

[11] J. Biazar and B. Ghanbari, "A new third-order family of nonlinear solvers for multiple roots," Computers and Mathematics with Applications, vol. 59, no. 10, pp. 3315-3319, 2010.

[12] J. R. Sharma and R. Sharma, "New third and fourth order nonlinear solvers for computing multiple roots," Applied Mathematics and Computation, vol. 217, no. 23, pp. 9756-9764, 2011.

[13] J. R. Sharma and R. Sharma, "Modified Chebyshev-Halley type method and its variants for computing multiple roots," Numerical Algorithms, vol. 61, no. 4, pp. 567-578, 2012.

[14] S. Kumar, V. Kanwar, and S. Singh, "On some modified families of multipoint iterative methods for multiple roots of nonlinear equations," Applied Mathematics and Computation, vol. 218, no. 14, pp. 7382-7394, 2012.

[15] W. Gautschi, Numerical Analysis: An Introduction, Birkhäuser, Boston, Mass, USA, 1997.

[16] S. Weerakoon and T. G. I. Fernando, "A variant of Newton's method with accelerated third-order convergence," Applied Mathematics Letters, vol. 13, no. 8, pp. 87-93, 2000.

[17] S. Wolfram, The Mathematica Book, Wolfram Media, Champaign, Ill, USA, 5th edition, 2003.
[18] A. Cayley, "The Newton-Fourier imaginary problem," The American Journal of Mathematics, vol. 2, no. 1, article 97, 1879.

[19] M. L. Sahari and I. Djellit, "Fractal Newton basins," Discrete Dynamics in Nature and Society, vol. 2006, Article ID 28756, 16 pages, 2006.

[20] J. L. Varona, "Graphic and numerical comparison between iterative methods," The Mathematical Intelligencer, vol. 24, no. 1, pp. 37-46, 2002.

[21] M. Scott, B. Neta, and C. Chun, "Basin attractors for various methods," Applied Mathematics and Computation, vol. 218, no. 6, pp. 2584-2599, 2011.

[22] B. Neta, M. Scott, and C. Chun, "Basin attractors for various methods for multiple roots," Applied Mathematics and Computation, vol. 218, no. 9, pp. 5043-5066, 2012. 


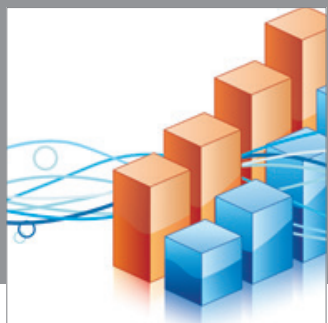

Advances in

Operations Research

mansans

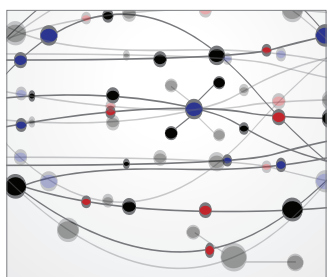

The Scientific World Journal
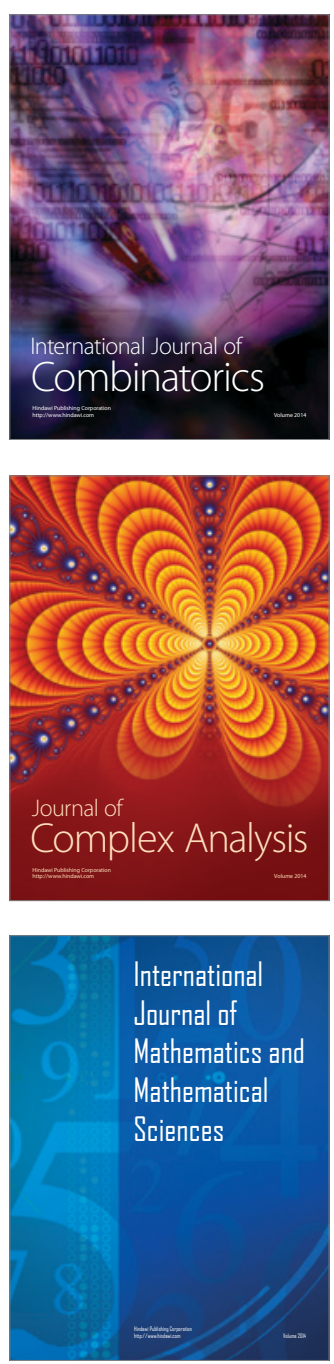
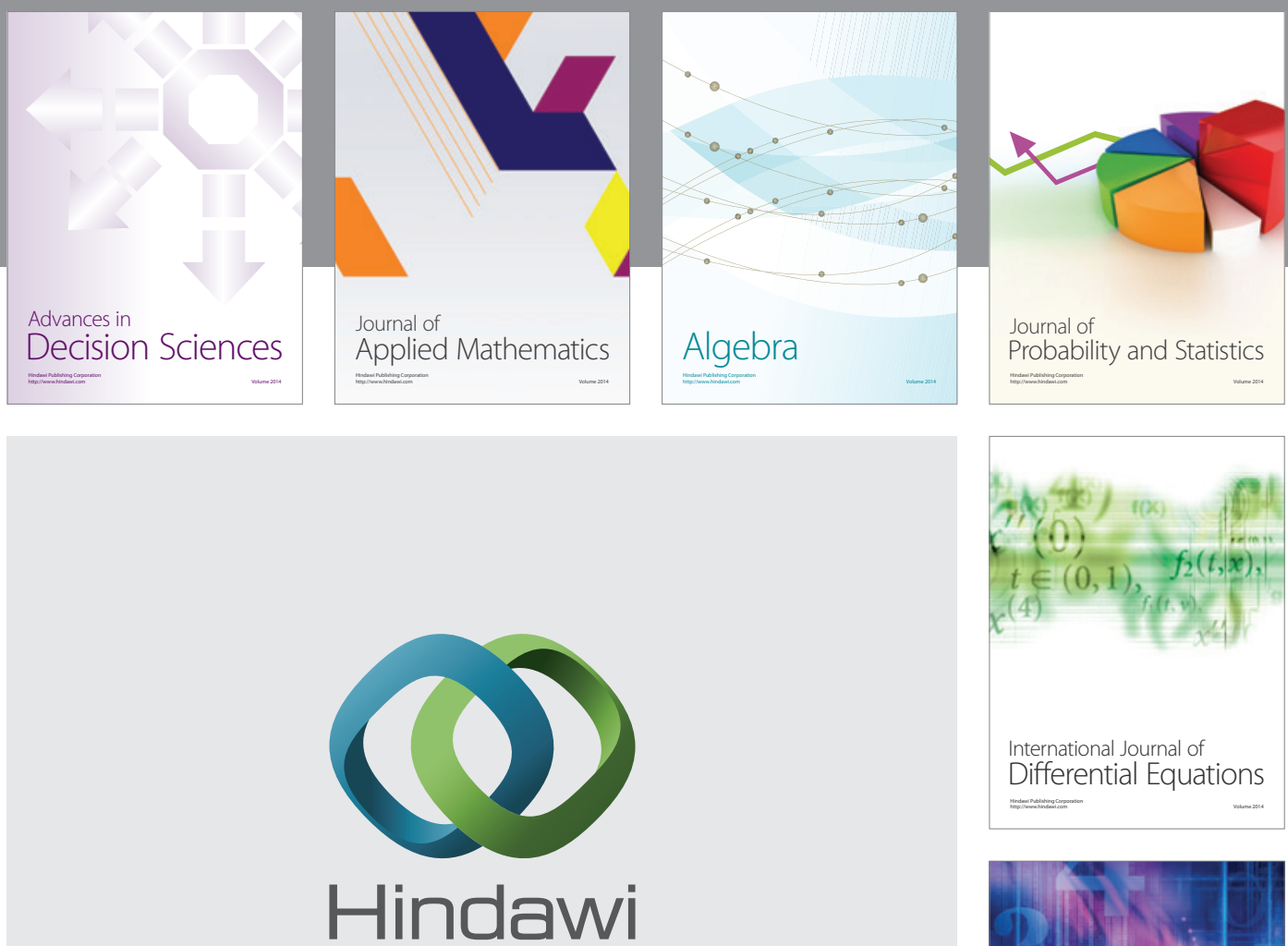

Submit your manuscripts at http://www.hindawi.com
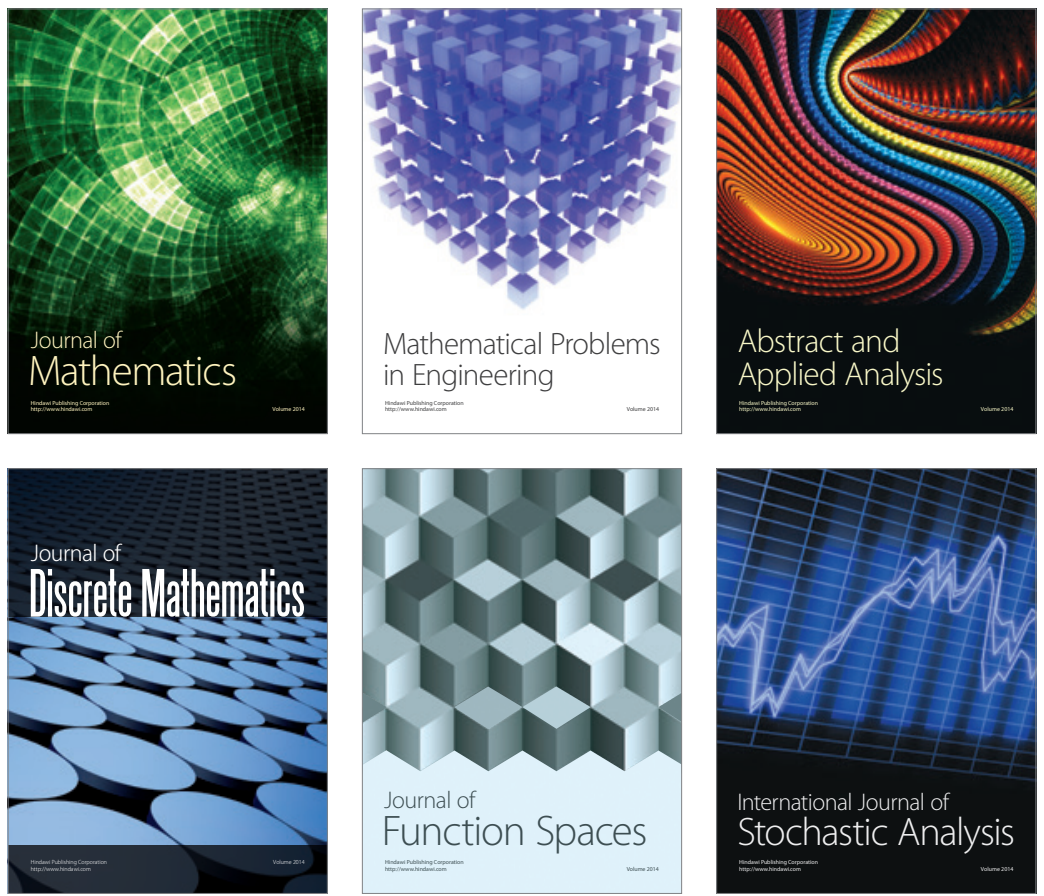

Journal of

Function Spaces

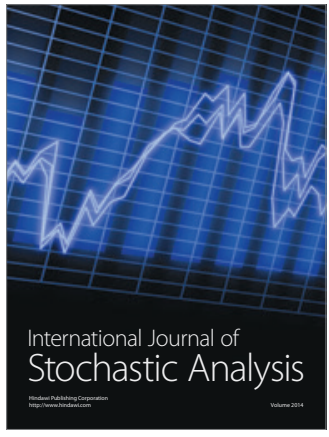

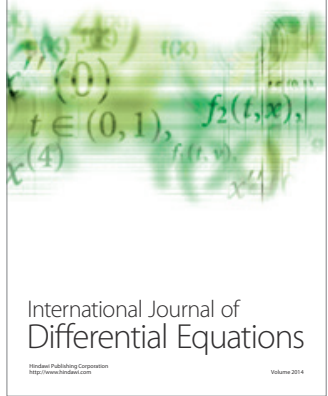
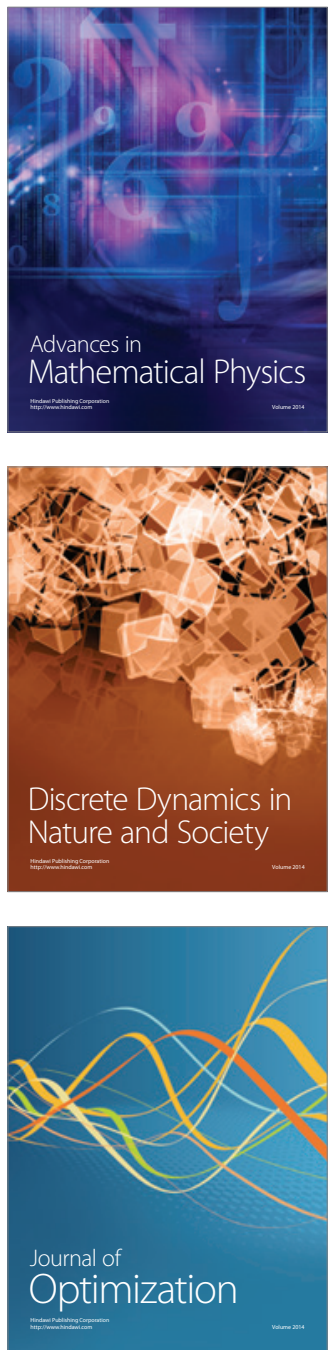